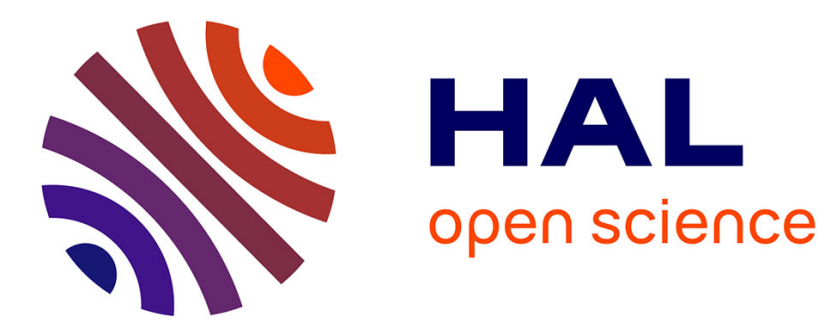

\title{
Study of Energy Saving in Carrier-Ethernet Network
}

\author{
Rihab Maaloul, Lamia Chaari Fourati, Bernard Cousin
}

\section{To cite this version:}

Rihab Maaloul, Lamia Chaari Fourati, Bernard Cousin. Study of Energy Saving in Carrier-Ethernet Network. Second Interantional Conference on Artificial Intelligence, Modelling and Simulations (AIMS 2014), Nov 2014, Madrid, Spain. 10.1109/AIMS.2014.30 . hal-01183313

\section{HAL Id: hal-01183313 https://hal.science/hal-01183313}

Submitted on 7 Aug 2015

HAL is a multi-disciplinary open access archive for the deposit and dissemination of scientific research documents, whether they are published or not. The documents may come from teaching and research institutions in France or abroad, or from public or private research centers.
L'archive ouverte pluridisciplinaire HAL, est destinée au dépôt et à la diffusion de documents scientifiques de niveau recherche, publiés ou non, émanant des établissements d'enseignement et de recherche français ou étrangers, des laboratoires publics ou privés. 


\section{Study of Energy Saving in Carrier-Ethernet Network}

\author{
Rihab Maaloul \\ National school of engineering, \\ Laboratory (LETI), \\ Sfax, Tunisia \\ mre7ab@gmail.com
}

\author{
Lamia Chaari Fourati \\ National school of engineering, \\ Laboratory (LETI), \\ Sfax, Tunisia \\ Lamia.Chaari@enis.rnu.tn
}

\author{
Bernard Cousin \\ University of Rennes 1 \\ (IRISA) \\ Rennes, France \\ Bernard.Cousin@irisa.fr
}

\begin{abstract}
Energy saving is a really interesting topic in today's networks. Indeed Information and Communication Technologies (ICT) systems are energy greedy, they require more and more energy and as energy is more and more expensive we seek to save it. Accordingly, energy saving has recently received considerable attention, as a promising approach for delivering ICT services with environmentally conscious use of technology by implementing energy aware strategies in networks infrastructures which lead to improve network resources utilization. Thus this paper addresses the issue of energy consumption in telecom networks and especially in Carrier-Ethernet networks. We review and describe relevant works currently proposed for energy-efficient operation of wired network infrastructure. Besides surveying some of the different technical issues that researchers are currently facing to reduce the energy consumption in networking, this paper identifies some of the remaining key research challenges that arise when such energy-saving techniques are extended for use in Carrier Ethernet networks.
\end{abstract}

Keywords: Green Networking, Energy Savings, Wired Networking, Carrier-Ethernet, IP Networks

\section{INTRODUCTION}

Reduction of unnecessary energy consumption in wired networks is becoming a main matter of public concern. Due to the impact of electricity consumption on the economic, environmental, and marketing fields, the energy saving in ICT sector and more specifically in networking is becoming a fundamental key issue. For example, nowadays networking infrastructures connecting data center to cloud afford high-capacity components and overprovisioning architectures to ensuring service availability which result in high power consumption. In addition, worldwide Internet Service Providers (ISPs) and Telecom operators have been confirmed in recent reports the rising price of energy [1]. These statements trigger many efforts which have been dedicated to cut unnecessary energy dissipation. Our objective is to provide a better understanding of several studies that have aimed to apply the energy saving for green networking. We can define the notion of green networking as the production of sufficient networking performance with minimum energy cost.

In networking energy saving may be achieved either by dynamically selecting the adequate paths which result in efficient use of energy or by switching off unnecessary links or network devices. In this paper, we focus on energy savings approaches devoted to be used in Carrier-Ethernet or in IP networks. Metro-Ethernet [2] is the use of Carrier-Ethernet technology in Metropolitan Area Networks (MANs). It can be used to connect business local Area Networks (LANs) and individual end users to Wide Area Networks (WAN) or to the Internet. Such use of Metro-Ethernet is to interconnect regional data centers or cloud consumers. Figure 1 presents the major segmentations of the network which are the access, aggregation (metro) and core networks, they are usually over dimensioned and need the implementation of energy aware mechanisms.

In literature there is few researches that are focused on Carrier-Ethernet energy saving mechanism. Since, Ethernet is massively deployed and it aims at reducing of both CAPEX and OPEX. So we expect a large room for energy saving in this network In order to better understand the possible ways to limit energy consumption in CarrierEthernet, we chose to overview in addition works related to energy saving on IP networks that could be adapted to Carrier-Ethernet.

The goal of the paper is to describe and to classify energy saving mechanisms proposed either in IP or in Carrier-Ethernet Networks and to discuss how can mechanisms applied in IP networks be adapted for Carrier-Ethernet. Energy saving mechanisms could be categorized into two categories: efficient path selection and powering off links and devices. In studied energy saving proposals, we focus on the optimization objective, on the network applicability and on main supported protocols and mechanisms for each proposal.

We have organized this paper into four main sections, one of which has sub-section. In the second section, we present the standards used in Carrier Ethernet context. In the third section, we present the main related methodologies. In the fourth section, we survey and classify energy aware approaches. Finally, we conclude this paper with a fifth section that discusses the importance and reveal the advantages and limitations of the studied approaches. Furthermore, we present our future work related to energy saving over Carrier-Ethernet network. 


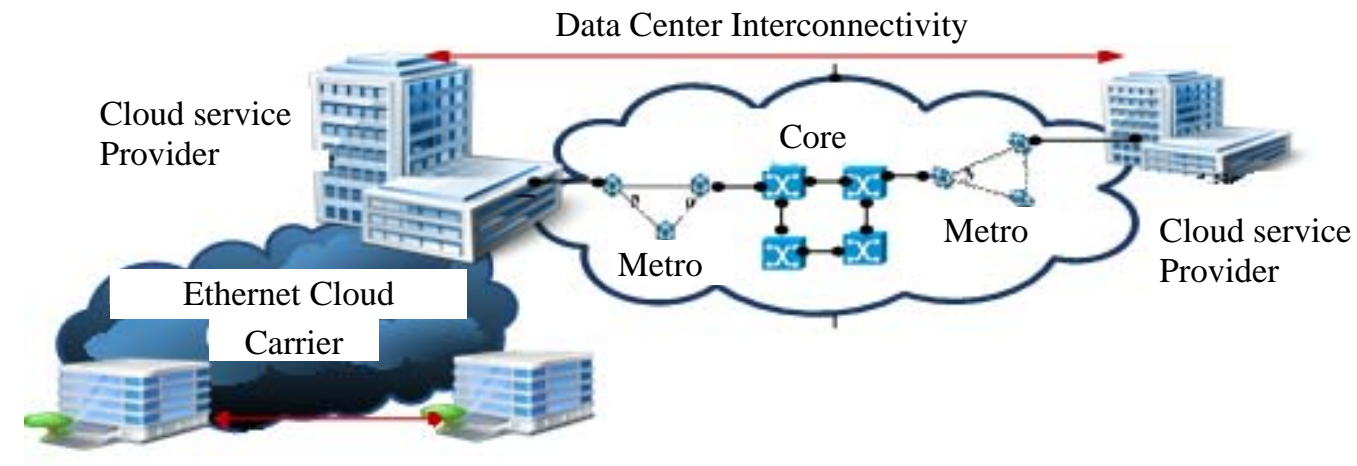

Cloud Business Interconnectivity

Figure 1. Network segmentation

II. CARRIER ETHERNET TECHNOLOGY AND STANDARDS

Incremental innovations are made within Ethernet standards. Therefore current services based on Ethernet technology are now used in MAN networks and Carrier Networks. One of the main reasons Ethernet is being widely deployed is due to its effective cost of both CAPEX and OPEX, when it is used by enterprises and ISPs.

The current Ethernet technologies rely on Spanning Tree Protocol (STP) standardized in IEEE 802.1D [1] and its variants: Rapid Spanning Tree Protocol (RSTP) [2] and Multiple Spanning Tree Protocol (MSTP) [3]. They manage the topology autonomously and provide a loop free connectivity across a variety of network nodes. Although these protocols have been used for most Ethernet networks, they are not powerful anymore to meet Metro Ethernet Network complexity. They have the following main shortcomings:

i) Inefficient use of resources: STP and its variants restrict the number of ports being used, which reduce the available bandwidth especially in case of high load traffic.

ii) Suboptimal path: the shortest path selection is based on the spanning tree for the entire network instead the shortest path between source/destination nodes.

iii) Re-convergence and Interruption: spanning trees implement the transactional distance-vector class of algorithm instead of a proper topology database, which adversely impacts the convergence time of an Ethernet network after a topology change [4].

Recently, a new class of shortest path routing solutions has been introduced for Ethernet networks, the Shortest Path Bridging (SPB), standardized in IEEE 802.1aq [5].

SPB aims to ensure frames forwarding on the shortest path within a Shortest Path Tree (SPT) region of a network by using an extension of IS-IS link state routing protocol [6]. In this way, SPB uses IS-IS procedures to construct and update the link state database in each SPT bridge.

Another alternative to STP like SPB is Transparent Interconnection of Lots of Links (TRILL) is an IETF standardized was introduced by Radia Perlman [9]. TRILL can be used in both current Ethernet networks and in IP Networks. Hence the denomination by Routing Bridge the devices which implement TRILL protocol.

\section{Related Methodologies}

There have been several approaches that aim to reduce power consumption in the area of communication networks. We have reviewed approaches that focus on energy saving in wired networks because our work is much more oriented toward power consumption of high performance Carrier Ethernet. A comprehensive taxonomy of green networking is provided in [1]. Authors have identified three categories of strategies: i) re-engineering, ii) dynamic adaptation, and iii) sleeping/standby. Reengineering aims at dimensioning and optimizing the network elements or devising completely new efficient equipment designs. Dynamic adaptation refers to modulating the capacities of packet processing engines and of network interfaces to meet actual requirements. The last category, sleeping/standby, considers the case where the network elements (or parts of) are switched off to a sleep state. All of these categories indicate that there is dependency between capacity and energy consumption.

A recent survey in [10] provides a comprehensive treatment of recent green networking techniques focusing on wired networking. They classify the existing research into four categories; i) adaptive link rate, ii) interface proxying, iii) energy-aware interfaces, and iv) energy-aware applications. Adaptive link rate category regroups approaches to reduce energy consumption by dynamically adapting the link rate to the traffic level. Interface proxying refers to attempts to "outsource" network operations from CPUs and such to low-power devices on, e.g., network interface card or in a case of a LAN to an external device, closer to the physical network. The third category regroups approaches related to the design and management of global network perspective. The last category, energy aware applications, calls for energy-efficient software designs.

Before attempting to minimize the energy consumption in networks, it is important to identify the sources of network power consumption and where the improvements could take place. In [11], authors argue that the architecture design can help to reduce the energy consumption in data center networking. In [12], authors consider a design approach for IP networks providing a possible way to achieve energy saving during the network design stage. Table I shows 
statistics foreseen in each segment, for the years of 2015-2020 according to [13].

TABLE 1. 2015-2020 NETWORK FORECAST/ DEVICE DENSITY AND ENERGY REQUIREMENTS IN THE BUSINESS-AS-USUAL CASE (BAU). EXAMPLE BASED ON THE ITALIAN NETWORK

\begin{tabular}{|c|c|c|c|}
\hline & $\begin{array}{c}\text { Power } \\
\text { consumption } \\
{[\mathbf{W}]}\end{array}$ & $\begin{array}{c}\text { Number of } \\
\text { devices } \\
{[\#]}\end{array}$ & $\begin{array}{c}\text { Overall } \\
\text { consumption } \\
\text { [GWh/year] }\end{array}$ \\
\hline Home & 10 & $17,500,000$ & 1,533 \\
\hline Access & 1,280 & 27,344 & 307 \\
\hline Metro & 6,000 & 1,750 & 92 \\
\hline Core & 10,000 & 175 & 15 \\
\hline
\end{tabular}

Overall network consumption 1,947

Also many standardized protocols such as Transparent Interconnection of Lots of links (TRILL) and Shortest Path Bridge (SPB), can be implemented in Carrier-Ethernet network to help in energy saving. An evaluation of TRILL (IETF) and SPB (IEEE 802.1aq) is presented in [14]. Both protocols compute the shortest path between any nodes pairs. They have a consistent view of all bridges, topology and location of end nodes due to the utilization of the IS-IS link state protocol. These protocols are as evolution of the Spanning Tree Protocol (STP) which is the Ethernet native routing algorithm. STP protocol can't adhere anymore to the requirements of Carrier-Ethernet complexity and innovation. It does not have information on the overall network topology that causes several shortcomings including a suboptimal path between node pairs and interruption and convergence due to any topology changes.

An optimization model through traffic engineering and employing Multiple Spanning Tree protocols is provided in [15]. This model considers the minimization of the energy consumption of Ethernet Carrier Network. [16] and [17], select the best energyefficient paths to deflect the traffic without powering off any equipment. These proposals are designed to improve the traffic engineering in Carrier-Ethernet by modifying the Spanning Tree Protocol STP and add new forwarding mechanisms.

In [18], an Energy Aware Routing algorithm is proposed to power off some network links during low traffic periods. The main idea of this algorithm is that only a subset of routers are elected as an exporters which evaluate their shortest path tree whereas the direct neighbors routers called importers routers utilize the shortest path tree of the exporters routers and power off the links that are no longer in new shortest path tree of the importers routers. Another approach in [19] puts routers in power saving mode during low traffic periods. In this regard, coordination is necessary for routers to enter in the sleeping state during nonpeak hours and to return into working state during peak hours.

\section{TAXONOMY OF ENERGY-AWARE STRATEGIES}

For the improvements on the network energy efficiency, a variety of strategies have been published. These strategies are being classified differently depending on the use of metrics and on the area of applications. Two main levels are considered to reduce the energy consumption: equipment level and the network level. At the equipment level, many techniques are proposed to optimize the network devices architecture such as the CMOS technology, circuit simplifications and the optimization of power management features (e.g, dynamic voltage scaling and adaptive link rate). Whereas at the network level, mechanisms are proposed to manage energy dissipation according to the network design and the network performance requirements (traffic volumes and QoS). Routing protocols, resource allocation strategies and Traffic Engineering approach are typical examples of mechanisms to the network level.

We give in this section a classification for the main energy saving strategies based on our literature review. The objective behind this classification is to study the energy efficiency strategies which could be adopted in Carrier Ethernet networks. We have classified these strategies into two mains categories: the first one selects the efficient paths which result in minimum energy consumption and the second one powers off devices or parts of them. Each category could be classified into sub-categories as is shown in the Figure 2. The first category comprises the strategies which focus on routing path selection subject to minimizing the energy consumption whether by selecting the shortest path between the source/destination pairs or by routing the traffic through energy efficient paths which are weighted according to their energy impacts. While the second category concern strategies that are acting on the power management of equipments networks.

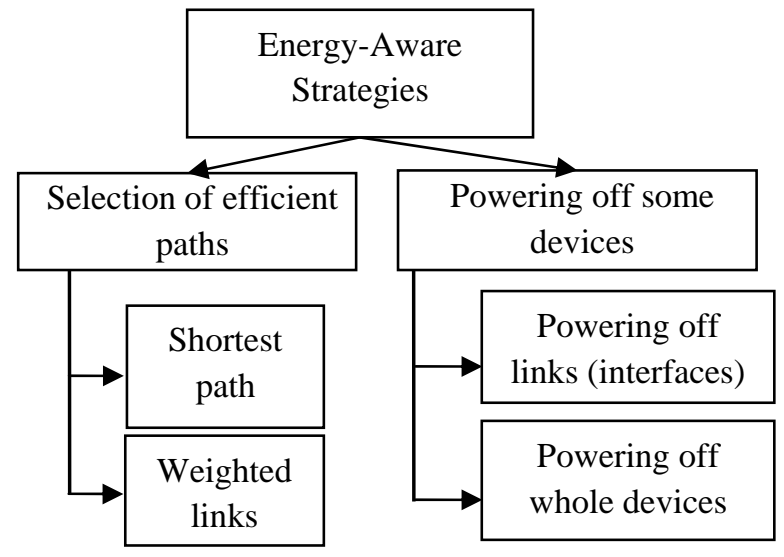

Figure 2. Taxonomy of Energy Aware Strategies

\section{A. The selection of efficient paths}

1) Shortest path: authors in [16] propose an improved version of the STP protocol named Shortcut Switching Strategy (SSS). This strategy uses some of the blocked links to forward traffic. Each node memorizes its blocking neighbor nodes (which are blocked by applying the STP protocol) in a table named BNNodes. If the destination address is in its BN-Nodes, it forwards the frame directly to that $\mathrm{BN}$-node via its blocked port. If the destination address is not in its BNNodes table, it forwards the frame as usual by using its 
traditional STP forwarding table. An example of SSS strategy is illustrated in Figure 3.

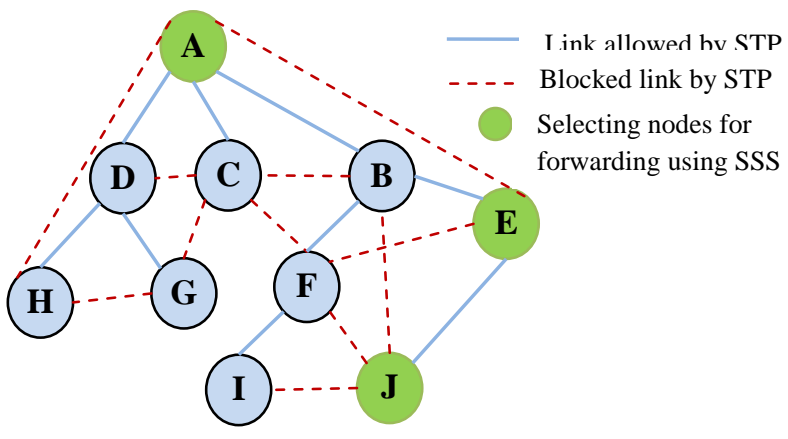

Figure 3. Example of SSS algorithm

Applying the SSS strategy, the number of hop in a path is decreased which improves the efficiency compared to STP by decreasing the routing cost and decreasing the average traffic volume on links and switches. Therefore it allows possible mitigation of traffic across the nodes which improve the load balancing on links. However, this strategy does not solve all the shortcomings of STP. In fact, switches lack information on the network topology so SSS is not efficient enough to handle a huge number of traffic.

The approach proposed in [17], belongs to the shortest path category, named HFS (Hierarchical Forwarding Strategy). This strategy consists of three major steps: i) Running standard STP, ii) Readdressing the nodes by using hierarchical addresses, iii) using new forwarding strategy. In this strategy, to reach a destination node each node selects the neighbor with lowest number of node counts as next hop. HFS enables a minimization of routing cost, thereby a potential minimization of transmission delay.

The standardized protocols SPB (IEEE 802.1aq) and TRILL fall in this category. Both select the shortest path between any two node pairs by using a slightly version of IS-IS link state routing protocol. TRILL and SPB allow load balancing, shortest path forwarding and in instant link failover.

2) Weighted links: authors in [13] compute the best path in terms of energy consumption for all traffic demand arrivals. Their algorithm computes the Kshortest paths for an incoming given traffic demand and evaluates the consumption impact of allocating such a demand on each network path, taking into account the traffic demands already allocated on the network. Then, the algorithm selects the path with minimum energy consumption impact on the network. This algorithm is compared with shortest path routing (i.e. $\mathrm{K}=1$-shortest path) and it is shown that important energy savings may be achieved, however at the expense of increasing the average number of hops per demand and the global network load as a consequence of using suboptimal (in terms of distance) paths.

Mentioned mechanisms that belong to the selection of efficient paths category can help in energy saving by introducing traffic engineering concepts. According to EEE (Energy Efficient Ethernet) [20], the energy efficiency is a function of the link utilization, packet transmission time, and the distribution of packet interarrival times. Proposals [11] and [12] try to satisfy these goals. So they contribute in energy saving without employing any techniques to change the mode power scheme. Furthermore, study in [11] shows that more the number of hop increases, more the energy consumption increases. This is generated by the power consumed by packet processor engines of network nodes.

\section{B. Powering off some equipments}

1) Powering off links (interfaces): In [18] a distributed energy saving routing algorithm, called EAR (Energy Aware Routing) is designed for IP networks. The main feature of EAR is that it is fully OSPF [21] compliant. It aimed at minimizing the active links of a network, i.e. the number of links to be used to route traffic. The scheme is to force a subset of routers to use some routes which are different from those elected in their SPTs (Shortest Path Trees). The set of network routers is divided into three subsets: exporters, importers, and neutral routers. This scheme is achieved by the three following phases: i) during the first phase, (Election of exporter routers), they calculate their shortest path trees (Dijkstra); ii) The second phase, called MPT(Modified Path Tree) evaluation, every importer routers use the exporter trees to compute their path trees and the links that have to be switched off; iii) Finally, each router computes its paths using Dijkastra algorithm on the residual network topology. The Figure 4 illustrates an example of a network graph in which the EAR was applied.

The SPTs computed through OSPF algorithm by node a, SPT (a), and node b, SPT (b) as are shown in Figure 4. Assuming that the node a is elected as an exporter router ER and then the node $\mathrm{b}$ is an importer router IR. The node $b$ has to perform MPT evaluation phase. It imports the SPT (a) as its own and then modifies it by inserting its self as root node, the result is as shown by MPT (b, a). When comparing SPT (b) and MPT (b, a), in terms of total number of links used to route traffic by node b, the result is that the link (b, f) is saved.
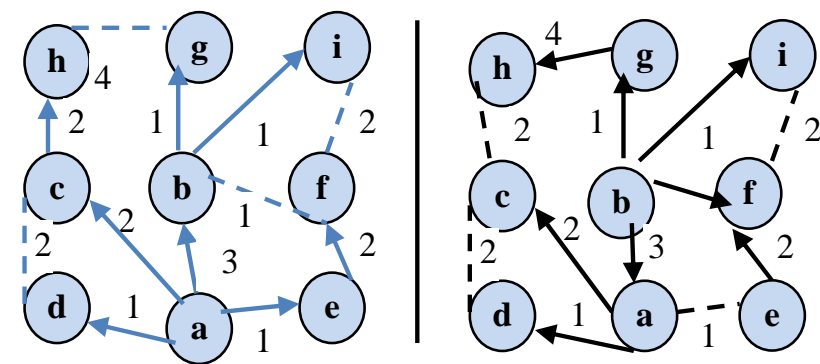

STP(a)

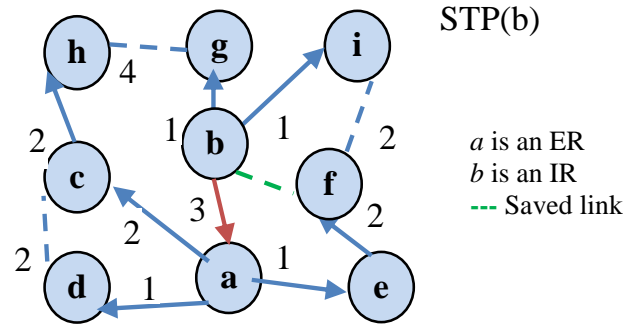

Figure 4. Network graph in which EAR is applied. 
2) Powering off some whole devices: In this subcategory, [19] proposes a distributed routing protocol, called GDRP (Green Distributed Routing Protocol), for power saving in wired core networks. This protocol is compatible with any existing distributed protocols such as OSPF. GDRP algorithm coordinates how routers in wired networks go into power saving mode without degrading quality of service and network connectivity. During peak hours the protocols will not degrade the network performance, while during non-peak hours a partial selection of routers can enter into sleep mode without degrading the quality of service and adversely affecting network connectivity. A power saving router has two states: working and sleeping (see Figure 5). In the working state, the routing operation is the same as a traditional router, but in the sleeping state the router enters sleep mode so that it will not process any packets until it switches back to the working state. The state of a router remains unchanged when the network is not loaded and it is in the sleeping state, or when the network is loaded and it is in the working state.

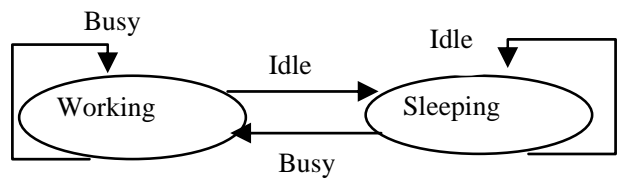

Figure.5. Transition states of power saving routers

A router estimates the network load by measuring the maximum utilization among all the links attached to itself (Umax). This is not the real overall network load but it is highly related to. When the Umax value of a router which is in a working state goes below a threshold, if the router computes the network connectivity can be maintained when the router will go in sleeping state, the router sends a request to the coordinator (which was previously elected among all routers) to get a permission to sleep. If the router gets a positive reply, then it broadcasts a new routing table and then goes to the sleeping state within a certain period. At the end of this sleeping period it will wake up and will listen the coordinator. When the Umax value of the coordinator is greater than a threshold, the router gets permission to switch to the working state. Otherwise it switches back to the sleeping state and sleep in another sleeping period.

In [22] and [23], two solutions are proposed using OpenFlow concept because it is flexible to manage and easy to deploy new protocols. Also it is able to coexist with carrier-class service.

The OpenFlow protocol is considered as the enabler of Software-Defined Networking (SDN) paradigm [24]. The basic SDN principle is the separation of data plane and control plane in networking devices (routers, switches). The data path portion remains residing on an OpenFlow switch, while decisions (such as putting link or devices on standby) are moved to a separate OpenFlow controller. The OpenFlow switch and controller communicate via the OpenFlow Protocol.
Unlike in router/switch where data path and control path (routing protocol) occur on the same device, these are separated in Open Flow [25].

In [22]authors propose a scheme which aims at putting some awaken nodes to sleep or awaking a sleeping nodes depending upon the traffic. This work uses OpenFlow protocol that has as main characteristic a centralized decision.

The controller periodically checks the traffic load in the network. If the traffic load is less than the capacity of the network, then it checks if any nodes can be put to sleep. If it can, it calculates the new route and put nodes to sleep. If the traffic load increases and the remaining active nodes cannot handle it, the controller wakes up the sleeping nodes and re-computes the routing path. This idea is achieved by the following steps: i) Measurement of the network traffic periodically, ii) Depending upon the network traffic, computation of the set of required nodes to forward the traffic and also determination of the root node. This later is selected in such a way that the maximum number of nodes can be put to sleep, iii) Use of the sleep/wake switching technique to put awake nodes to sleep or wake sleeping nodes as required. iv) Change of the routing path using the awake nodes. This step uses the Shortest Path First algorithm (Dijkstra) to compute the routing paths.

In [15] an optimization model has been proposed aimed at minimizing the energy consumption in Carrier-Ethernet networks. This model uses Multiple Spanning Tree Protocol (MSTP) IEEE 802.1s. This object is performed in such a way that a portion of the network is forced by the objective function of the model to remain unused, thus making it possible to turn off the elements of that portion of the network, which are put into sleep mode to conserve energy. This leads to use the fewest possible nodes and arcs, while continuing to ensure full connectivity and the management of all requests. A trade-off between the minimization of the energy consumption and the load balancing objective is also proposed, that help to maintain the network performance.

A summary of proposals that use in green networking is provided in Table II. It lists the optimization objective, the applicable part network (network type) and mechanisms for each proposal.

\section{CONCLUSION AND FUTUR WORKS}

This paper reviewed the relevant means that have been used to reduce the energy consumption in telecom networks domains. Our major concern is studying how to improve energy efficiency in Carrier Ethernet networks. In this regard, we described the Carrier Ethernet technology, its standards and the network segmentation. Moreover we presented the importance of such topic and proposed a classification of main related works. We have identified the causes and the different level of the energy consumption problem in networking.

For the proposals of the first category where all the network elements are always powered on, we observe that they do not give a considerable energy gain. While they contribute to ensure an efficient use of resources 
which is in turn help to avoid energy dissipation and they satisfy QoS and performance constraints.

The second category regroups proposals to reduce energy consumption by powering off some whole nodes or line cards. This class of solution offers an achievable energy gain.

Studies approach designed for IP networks could be adopted in Carrier-Ethernet network by applying protocols that are able to run directly over it and handling MAC addresses.

Our future work will include advanced investigation for energy saving in Carrier-Ethernet. In this context, we will focalizing our researches on powering off line cards (interfaces) mechanisms and as a first contribution we propose to modify EAR algorithm [18], which is designed to IP network, making it Carrier-Ethernet compliant. As described earlier the main feature of EAR is its OSPF compliance, so it can be easily integrated into SPB protocol. Seeing that SPB uses IS-IS protocol and both IS-IS and OSPF behave similarly except IS-IS is able to handle both MAC and IP addresses.

In fact, EAR provides energy saving during light utilization periods (low traffic hours) by turning off a set of links and rerouting the paths crossing them in order to minimize the energy requirements, while meeting the operational constraints and current switching workloads. Furthermore, the results obtained by EAR showed that more than $30 \%$ off links can be potentially turned off which encourage us to investigate and to adapt EAR for Carrier-Ethernet.

We consider the limitation of EAR that it is unaware of traffic conditions. Even in [26], the authors proposed an enhanced version of EAR strategy that overcomes the limitations of the old EAR solution [18] by defining the concepts of "move" that permit to early evaluate all the positive and negative effects of an exportation and can modulate the network performance and allows a QoS strategy. The enhanced version presents a significant complexity and poor performance in some cases.

Another direction of future work include the implementation of OpenFlow over Carrier Ethernet Switch Router motivated by [23] to conceive an energy aware mechanisms dedicated to Carrier Ethernet according the SDN paradigm.

TABLE II ENERGY AWARE PROPOSALS COMPARISON

\begin{tabular}{|c|c|c|c|c|}
\hline & Proposal & Optimization Objectives & Applicable Part & Mechanisms \\
\hline \multirow{3}{*}{ 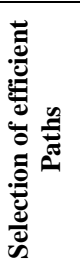 } & [16] & $\begin{array}{l}\text {-Efficiency Utilization of Switches and links } \\
\text {-Minimize path hops }\end{array}$ & $\begin{array}{c}\text { Carrier Ethernet Network } \\
\text { (Core and Metro segments) }\end{array}$ & -Modified STP (IEEE 802.1D) Protocol \\
\hline & {$[17]$} & -Minimize the number of hop count & $\begin{array}{l}\text { Carrier Ethernet Network } \\
\text { (Core and Metro segments) }\end{array}$ & $\begin{array}{l}\text {-Modified STP Protocol and } \\
\text { Hierarchical Forwarding Strategy } \\
\text {-Traffic Engineering }\end{array}$ \\
\hline & [13] & $\begin{array}{l}\text {-Reduction of Routing Cost considering the } \\
\text { Energy consumption }\end{array}$ & $\begin{array}{l}\text { Ethernet Network } \\
\text { (Metro and Access } \\
\text { segments) }\end{array}$ & $\begin{array}{l}\text { Flow Allocation Algorithm considering } \\
\text { the energy consumption of incoming } \\
\text { flow }\end{array}$ \\
\hline \multirow{5}{*}{ 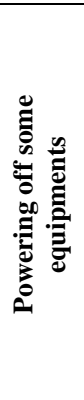 } & [18] & -Pwering off some network links & $\begin{array}{c}\text { IP Network } \\
\text { (Metro and core segments) }\end{array}$ & $\begin{array}{c}\text {-Heuristic (OSPF based) } \\
\text {-Linear Programming }\end{array}$ \\
\hline & [19] & $\begin{array}{l}\text {-Putting some routers into sleep mode } \\
\text {-Maintain the QoS and network connectivity }\end{array}$ & $\begin{array}{c}\text { IP Netwok } \\
\text { (Metro and core segments) }\end{array}$ & $\begin{array}{l}\text {-Heuristic (General Distributed } \\
\text { Protocols based) }\end{array}$ \\
\hline & {$[20]$} & $\begin{array}{l}\text {-Putting links in low power mode } \\
\text {-Meeting the traffic requirements }\end{array}$ & $\begin{array}{l}\text { Ethernet Network } \\
\text { (Acces segments) }\end{array}$ & -Packet coalescing technique \\
\hline & [15] & $\begin{array}{l}\text {-Powering off nodes } \\
\text {-Load balancing }\end{array}$ & $\begin{array}{l}\text { Metro Ethernet Network } \\
\text { (Metro and core segments) }\end{array}$ & $\begin{array}{c}\text {-Heuristic } \\
\text {-Linear Programming }\end{array}$ \\
\hline & [ 22] & $\begin{array}{l}\text { - Putting some Switches/Routers to sleep } \\
\text {-Maintain the QoS and network connectivity }\end{array}$ & $\begin{array}{l}\text { Open Flow Network } \\
\text { (Any segments) }\end{array}$ & $\begin{array}{l}\text {-Soft Defined Network approach } \\
\text {-Controller configuration and traffic } \\
\text { engineering }\end{array}$ \\
\hline
\end{tabular}




\section{REFERENCES}

[1] R. Bolla, R. Bruschi, F. Davoli, and F. Cucchietti. Energy effciency in the future internet: "A survey of existing approaches and trends in energy-aware fixed network infrastructures”, IEEE Communications Surveys \& Tutorials, Volume 13, Issue: 2, 2011.

[2] A. Achim, K. Andreas, Siemens Networks GmbH\&Co KG, Germany, "Carrier Grade Metro Ethernet Networks", Photonic Networks, ITG Symposium on, 2007.

[3] IEEE Standard for Local and Metropolitan Area Networks. Media Access Control (MAC) Bridges. IEEE Standard 802.1D-1998, 1998.

[4] IEEE Amendment 2: Rapid Reconfiguration Amendment to IEEE Std 802.1D, 1998 Edition. IEEE Std 802.1w-2001.

[5] IEEE Amendment 3: Multiple Spanning Trees Amendment to IEEE Std 802.1Q ${ }^{\mathrm{TM}}$, 1998 Edition. IEEE Std 802.1s2002

[6] D. Allan, P. Ashwood-Smith, N. Bragg, J. Farkas, D. Fedyk, M. Ouellete, M. Seaman, P.Unbehagen, "Shortest Path Bridging: Efficient Control of Larger Ethernet Networks”, IEEE Communications Magazine, pages: 128135, Volume:48, Issue: 10 Octobre 2010.

[7] Interworking Task Group of IEEE 802.1, IEEE P802.1aq Draft Standard for Local and Metropolitan Area Networks - Media Access Control (MAC) Bridges and Virtual Bridged Local Area Networks, 2011.

[8] R.Callon, "Use of OSI IS-IS for routing in TCP/IP and dual environments,” RFC 1195, December 1990.

[9] R. Perlman, Intel Labs, and Donald Eastlake, Huawei technolgies, “The Internet Protocol Forum”, 2011, http://www.ipjforum.org/?p=582

[10] A. Bianzino, C. Chaudet, D. Rossi, and J. Rougier. “A survey of green networking research". Communications Surveys Tutorials, IEEE, 14 (1), 3-20, 2012.

[11] L. Gyarmati and T. Anh Trinh, "How can architecture help to reduce energy consumption in data center networking? ” e-Energy, page 183-186. ACM, (2010)

[12] G. Shen and R. S. Tucker, "Energy-minimized design for IP over WDM networks," IEEE/OSA J. Optical Communication Network, vol. 1, no. 1, pp. 176- 186, June 2009.

[13] I. Seoane, J.A. Hern'andez, P. Reviriego, and D. Larrabeiti, "Energy-aware flow allocation algorithm for Energy Efficient Ethernet networks”, Software Telecommunications and Computer Networks (SoftCOM), 15-17 Sept 2011.

[14] J. Soeurt and I. Hoogendoorn, "Shortest path forwarding using OpenFlow", University of Amsterdam, February 13 2012. Report, available at http://www.delaat.net/rp/20112012/p25/report.pdf

[15] D. Corti, A. Capone and B. Sanso, "Minimizing the Energy Consumption of Carrier Grade Ethernet with Multiple Spanning Trees”, In (WoWMoM) / (IEEE), June 2011.

[16] F. Faghani, G. Mirjalily and F.Hendassi, "Shortcut Switching Strategy in Metro Ethernet Networks", Computer Communications journal Volume 34 Issue 8, June, 2011, Pages 1022-1032.

[17] F. Faghani, G.Mirjalily and F. Hendassi, "New Forwarding Strategy for Metro Ethernet Networks Based on Hierarchical Addressing”,14th International Conference on “Advanced Communication Technology”, (ICACT), 2012 Page(s): 721 - 7252012.

[18] A. Cianfrani, V. Eramo, M. Listanti, Marco Marazza, Enrico Vittorini, “An energy saving routing algorithm for a green OSPF protocol”, IEEE INFOCOM, 10-15 march, 2010, SAN Diago, USA.

[19] K. Ho and C. Cheung. "Green distributed routing protocol for sleep coordination in wired core networks". In IEEE 6th International Conference on Networked Computing, Gyeongju, Korea (South), May 2010.
[20] K. Christensen et al, "IEEE 802.3az: The Road to Energy Efficient Ethernet”, IEEE Communications Magazine, November 2010.

[21] J. Moy, S. Murphy, M. Yeung and Z. Zhang, "OSPF Version 2,” RFC 2328, April 1998.

[22] B. Bahadur Bista, M. Takanohashi, T. Takata, and D. Rawat, "A Power Saving Scheme for Open Flow Network”, Journal of Clean Energy Technologies, Vol. 1, No. 4, October 2013.

[23] C. Vaishampayan, S. Bidkar, S. Mehta, and D. Bhamare "Demonstrating OpenFlow over Carrier Ethernet Router (CESR) - Aservice Perspective”, 2012.

[24] Cisco and its affiliates, "Software-Defined Networking: Why We Like It and How We Are Building On It”, white paper, 2013.

[25] OpenFLow Switch Specification. [Online]. Available: http://www.openflow.org/wp/documents/.

[26] A. Cianfrani, V. Eramo, M. Listanti, and M. Polverini , "An OSPF Enhancement for energy saving in IP Networks", workshop on green communication and networking, Infocom 2011, Shanghai, China during April 10-15. 